\title{
A Hybrid Prediction Method of Thermal Extension Error for Boring Machine Based on PCA and LS-SVM
}

\author{
QiangCheng ${ }^{1}$ Yanwei $\mathrm{Yu}^{2}$, Guangpeng $\mathrm{Li}^{1}$, WeishuoLi ${ }^{1}$ Bingwei Sun ${ }^{1}$ and Ligang Cai ${ }^{1}$ \\ ${ }^{1}$ College of Mechanical Engineering and Applied Electronics Technology,Beijing University of Technology, Beijing 100124, China \\ ${ }^{2}$ Henan Mechanical and electrical Vocational college ,Zhengzhou 451191, China
}

\begin{abstract}
Thermal extension error of boring bar in z-axis is one of the key factors that have a bad influence on the machining accuracy of boring machine, so how to exactly establish the relationship between the thermal extension length and temperature and predict the changing rule of thermal error are the premise of thermal extension error compensation. In this paper, a prediction method of thermal extension length of boring bar in boring machine is proposed based on principal component analysis (PCA) and least squares support vector machine (LS-SVM) model. In order to avoid the multiple correlation and coupling among the great amount temperature input variables, firstly, PCA is introduced to extract the principal components of temperature data samples. Then, LS-SVM is used to predict the changing tendency of the thermally induced thermal extension error of boring bar. Finally, experiments are conducted on a boring machine, the application results show that Boring bar axial thermal elongation error residual value dropped below $5 \mu \mathrm{m}$ and minimum residual error is only $0.5 \mu \mathrm{m}$. This method not only effectively improve the efficiency of the temperature data acquisition and analysis, and improve the modeling accuracy and robustness.
\end{abstract}

\section{Introduction}

In recent years, with the continuous development of equipment manufacturing industry, higher and higher requirement of precision is presented to machine tools [1]. For boring machine, thermal error of the boring bar is one of the key error sources [2-3], and research shows that the thermal error of boring machine can accounts for $40 \%$ $70 \%$ of total errors[4-5].In general, the methods to reduce the thermal errors can be classified into error prevent method and error compensation method [6]. Error prevention refers that the thermally induced errors could be reduced with structure improvement of the machine tool itself through design and manufacturing technology. In the process of implementation, however, there are many physical limits, cannot completely overcome design difficulties [7-8]. As a result, the thermal error compensation technology is an effective and economical method of reduce the thermal error of machine tool $[9-10]$.

Generally speaking, in order to get a high precision and good robustness of thermal error model, it is important to obtain the temperature data of each site and the corresponding heat deformation in the processing. However, the boring machine temperature field is nonlinear, degeneration and the extremely complex distribution, which requires layout of a large number of temperature sensors to obtain more accurate temperature field on boring machine. Arrangement of much temperature sensors can lead to the problem of dealing with the coupling mong a large amount of data, and at the same time much wiring will also affect the machining process. Therefore, many scholars carried on the thorough research in terms of temperature measuring point optimization and achieved some results. Yang et al. proposed a method based on thermal error sensitivity figure, however this method needs to select more measuring points to ensure accuracy and otherwise the efficiency of modeling will be reduced [11]. Wang et al. proposedPSO optimization $\mathrm{K}$ mean clustering method to optimize the temperature measurement points. The method can improve the speed of modeling, but the random initial values may lead to different clustering results, and even no solution [12]. Luo et al. offered a method based on the stepwise linear regression, which provides a new way for thermal error modeling [13]. According to the thermal error and the temperature field of the dynamic, Ma optimized the temperature measurement point [14]. Lee of South Korea has been reduced temperature measurement point by using correlation coefficient and linear regression method. Some results have been obtained [15], but the nonlinear of temperature data is not considered adequately. Creighton et al. established the model of temperature rise and thermal deformation by using finite element method[16].The temperature rise and thermal deformation of the key points were obtained by using thermocouple and capacitance meter. The optimized process is relatively straightforward, but the boundary conditions of finite element method are not uniform. Han et al. proposed a 
correlation coefficient analysis and fuzzy $\mathrm{C}$ means clustering method to reduce the number of temperature sensors, the number of sensors from 32 to 5 [17]. However, this method has the following disadvantages: in order to select the appropriate temperature measurement point must collect a large number of temperature data, the available data must be typical distribution, such as normal (Gaussian) distribution.

It can be seen that in the process of the thermal error modeling and compensation, one of the key is to reduce the large number of temperature measuring points. Therefore, PCA method is proposed to reduce temperature measuring point in this paper, and then LS-SVM modeling method is used to predict the thermal error of boring machine. In order to optimize temperature measuring points and realize accurate prediction of thermal extension error, this paper is arranged as following. The section 2 introduces the reduction method of PCA. Section 3 introduces the relation prediction of the error and temperature based on LS-SVM method. In Section 4, a CNC boring machine is selected as an example to verify the method. The conclusions are presented in Section 5 .

\section{Reduction the dimension temperature data based on PCA}

Theoretically, thermal extension error of boring baris mainly influenced by the whole boring machine temperature changes. In order to establish the accurate thermal extension error of boring bar prediction model, it is required that all parts on the boring machine are installed a large number of temperature sensors to measure real-time temperature during boring machining. Generally speaking, the temperature measuring point selecting from several to several hundreds [18]. However, too much temperature measuring point not only increase the workload, but also increase the mutual coupling between the measured data, which may affect the accuracy of prediction. In order to reduce the amount of input and improve the speed and accuracy of modeling, therefore, in this section a boring machine showed in Fig. 1 is selected as an illustrative object to apply PCA to reduce the temperature measuring points.
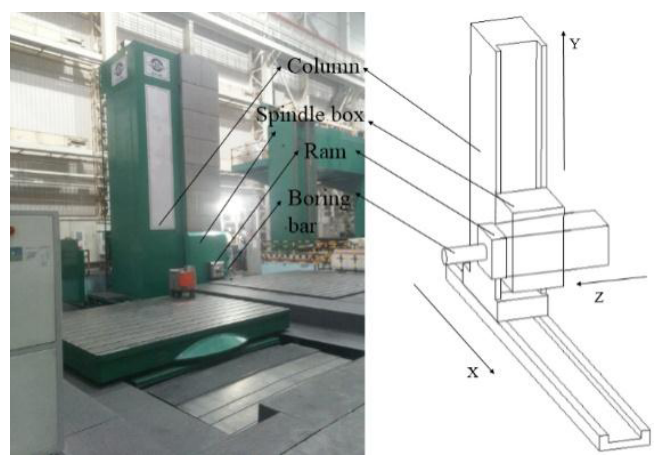

Figure 1. Structure diagram of the illustrative boring machine

PCA aims to use the ideas of dimension reduction to make the majority of indicators transform into a few comprehensive indexes [19-20].The main idea is to find the spindle direction of the data and constitute a new coordinate system spindle, the dimension of the new coordinate system can be lower than that of the original dimension, then the data are mapped to the new coordinate system from the original coordinate system. This mapping process is the process of feature data dimension reduction. So, PCA is used to reduce the dimension of the temperature data, the specific steps are as follows:

(1) Firstly, sort out a lot of boring machine temperature data to construct matrix $\mathbf{T}_{m n}$ Where, $m$ is the number of temperature data collection sample, $n$ is the number of temperature sensors installed on the boring machine.

$$
T_{m \times n}=\left[\begin{array}{cccc}
t_{11} & t_{12} & \cdots & t_{1 n} \\
t_{21} & t_{22} & \cdots & t_{2 n} \\
\vdots & \vdots & & \vdots \\
t_{m 1} & t_{m 2} & \cdots & t_{m n}
\end{array}\right]
$$

(2) Secondly, standardize the elements in temperature matrix according to Eq.(2).

$$
\left\{\begin{array}{l}
T_{i j}^{*}=\left(T_{i j}-\overline{T_{j}}\right) / \operatorname{std}\left(T_{j}\right) \\
\overline{T_{j}}=\frac{1}{M} \sum_{i=1}^{M} T_{i j} \\
\operatorname{Std}\left(T_{j}\right)=\sqrt{\frac{1}{M} \sum_{i=1}^{M}\left(T_{i j}-\overline{T_{j}}\right)^{2}}
\end{array}\right.
$$

In which, $i=1,2, \cdots, \mathrm{m}, \mathrm{j}=1,2, \cdots n ; \overline{T_{j}}$ is the mean value of the $j$ column temperature, and $\operatorname{Std}_{\left(T_{j}\right)}$ is the standard deviation of the $j$ column temperature.

(3)Thirdly, calculate the covariance matrix ( $S$ )of the standardization data $F_{i j}{ }^{*}$ by Eq.(3).

$$
\begin{aligned}
& S_{j k}=\frac{1}{M} \sum_{i=1}^{M}\left(T_{i j}^{*}-\bar{T}_{j}\right)\left(T_{i k}^{*}-\bar{T}_{k}\right) \\
& j=1,2, \cdots, n ; k=1,2, \cdots, n ;
\end{aligned}
$$

(4)Fourthly, calculate the eigenvalues of the covariance matrix $S$ and order by size, $\lambda_{1} \geq \lambda_{2} \geq \lambda_{3} \geq \cdots \lambda_{n}$; The eigenvalue of the corresponding eigenvector are $\boldsymbol{\alpha}_{1}, \boldsymbol{\alpha}_{2}, \boldsymbol{\alpha}_{3}, \cdots \boldsymbol{\alpha}_{n}$.

(5)Finally, by the eigenvalues of the covariance matrix $S$, compute variance contribution rate $\beta_{k}$ and the cumulative variance contribution rate $\beta(k)$ is obtained as:

$$
\left\{\begin{array}{l}
\beta_{k}=100 \% \lambda_{k} / \sum_{k=1}^{M} \lambda_{k} \\
\beta(k)=\sum_{q=1}^{k} \lambda_{q} / \sum_{q=1}^{M} \lambda_{q}
\end{array}\right.
$$

The number of principal components which needed to be extracted is determined by the principle that the cumulative variance contribution rate is more than $85 \%$, and the principal component of $\boldsymbol{A}_{\boldsymbol{i}}$ can be expressed as following: 


$$
A_{i}=T_{i j}^{*} \boldsymbol{\alpha}_{i}
$$

In which, $i=1,2, \cdots, q ; q$ is the number of the principal components.

\section{Relation prediction of the error and temperature}

In machine learning field, SVM is a supervised learning model, which is usually used for pattern recognition, classification, and regression analysis [21].The basic idea is to map a given sample set $T=\left\{\left(x_{i}, y_{i}\right) \mid x_{i} \in R^{n}, y_{i} \in(-1,+1)\right\}_{i=1}^{n}$ to a high dimensional feature space by nonlinear kernel function that meets Mercer condition, and construct an optimal classification hyper-plane, which can separate two classes of data points as far as possible.

Training sample $\left(x_{i}, y_{i}\right)$ is given, in which, $i=1,2, \cdots, n, x \in R^{d}, \mathrm{y} \in \mathrm{R} . x_{i}$ is training sample input for $\mathrm{n}$ dimension, $y_{i}$ is training sample output for $\mathrm{n}$ dimension .Decision function is $f(x, w)=w \phi(x)+b$. So, the training of the model is transformed into the structural risk minimization. The mapping function of the original space sample to high dimensional feature space is $\phi(t), W$ is the weight vector, $x=\left(T_{1}, T_{2}, T_{3}, \cdots, T_{n}\right)$ is composed of $N$ boring machine temperature vector, and $b$ is a threshold. Based on the principle of structural risk minimization, $w$ and $b$ are obtained by minimizing the structural risk function $J=\|w\|^{2} / 2+\mathrm{s} E$; Where, $s$ is the regularization parameter, $E$ is error loss function.LS-SVM is developed on the basis of SVM, and the estimation of the model function can be described as the optimization of $J$ function:

$$
\left\{\begin{array}{c}
\min J(w, \xi)=\frac{1}{2} w^{T} w+\frac{1}{2} s \sum_{i=1}^{n} \xi_{i}^{2} \\
\text { s.t. } \quad y_{i}=w^{T} \varphi\left(x_{i}\right)+b+\xi_{i} \\
\xi_{i} \geq 0 \quad i=1,2, \cdots, n
\end{array}\right.
$$

In order to convert the Eq. (4) to the dual problem, the Lagrange function is introduced as follows:

$$
\begin{aligned}
& L(w, b, \boldsymbol{\xi}, \boldsymbol{\alpha})=\frac{1}{2} w^{T} w+\frac{1}{2} s \sum_{i=1}^{n} \xi_{i}^{2}- \\
& \sum_{i=1}^{n} \alpha_{i}\left[w^{T} \varphi\left(t_{i}\right)+b+\xi_{i}-y_{i}\right]
\end{aligned}
$$

In the formula, $\alpha_{i}=\left(\alpha_{1}, \alpha_{2}, \cdots, \alpha_{3}\right)$ is the Lagrange multiplier; $w, b$ is model parameters; $\xi$ is training set prediction error vector. According to the necessary condition of extreme value, partial derivative of the Eq. (5) is calculated as follows:

$$
\left\{\begin{array}{l}
\frac{\partial L}{\partial w}=0 \rightarrow w=\sum_{i=1}^{n} \boldsymbol{\alpha}_{i} \varphi\left(x_{i}\right) \\
\frac{\partial L}{\partial b}=0 \rightarrow \sum_{i=1}^{n} \boldsymbol{\alpha}_{i}=0 \\
\frac{\partial L}{\partial \boldsymbol{\xi}_{i}}=0 \rightarrow \boldsymbol{\alpha}_{i}=2 s \boldsymbol{\xi}_{i} \\
\frac{\partial L}{\partial \boldsymbol{\alpha}_{i}}=0 \rightarrow w^{T} \cdot \varphi\left(x_{i}\right)+b+\boldsymbol{\xi}_{i}-y_{i}=0
\end{array}\right.
$$

The optimization problem is transformed into solving linear equation Group(6).

$$
\left\{\begin{array}{l}
\left(\begin{array}{llll}
0 & 1 & \cdots & 1 \\
1 & A & \cdots & B \\
\vdots & \vdots & & \vdots \\
1 & B & \cdots & A
\end{array}\right)\left(\begin{array}{c}
b \\
\alpha_{1} \\
\vdots \\
\alpha_{i}
\end{array}\right)=\left(\begin{array}{c}
0 \\
y_{1} \\
\vdots \\
y_{i}
\end{array}\right) \\
\left\{\begin{array}{l}
A=\varphi\left(x_{1}\right) \varphi\left(x_{i}\right)+\frac{1}{s} \\
B=\varphi\left(x_{i}\right) \varphi\left(x_{1}\right)
\end{array}\right.
\end{array}\right.
$$

According to the Mercer conditions, the Gauss kernel function $K\left(x, x_{i}\right)$ is selected to as kernel function. So, LS-SVM regression modeling can be expressed as follows:

$$
f(x)=\sum_{i=1}^{n} \alpha_{i} K\left(x, x_{i}\right)+b
$$

The kernel function can be chosen to satisfy the symmetric function of Mercer condition, commonly kernel function has linear kernel function and polynomial kernel function and Gaussian kernel function. In this paper, RBF core $K\left(x, x_{i}\right)=\exp \left[-\left\|x-x_{i}\right\|^{2} /\left(2 \sigma^{2}\right)\right]$ is selected; So that the least squares support vector machine estimation algorithm contains two sensitive parameters: regularization parameter $s$ and kernel function parameter $\sigma$, and these two parameters can be obtained by cross identification (CV)[22-23].

\section{Experimental analysis and model- ing verification}

\subsection{Experimental data acquisition}

The boring bar system structure of machine tool is as shown in the Fig. 2, in which the spindle box is mainly composed of three parts, including a static pressure radial bearing, a gear box, and a spindle motor. The gear box was divided into two grades, the higher speed was $2000 \mathrm{r} / \mathrm{min}$, and the lower onewas600r/min. Experiment was divided into two parts. Firstly, the gear box was set to be $600 \mathrm{r} / \mathrm{min}$, and the purpose was to make the natural extension of boring bar to reach the thermal equilibrium state, and the spindle was stopped in order to make the boring bar cool naturally until the length of the boring bar was no longer significantly reduced. In Second part, the gear box was set to be $2000 \mathrm{r} / \mathrm{min}$, and the above process was repeated. The sensors were mounted as shown in Fig. 3 and Table 1, and the temperature values were read by the numerical control system. 


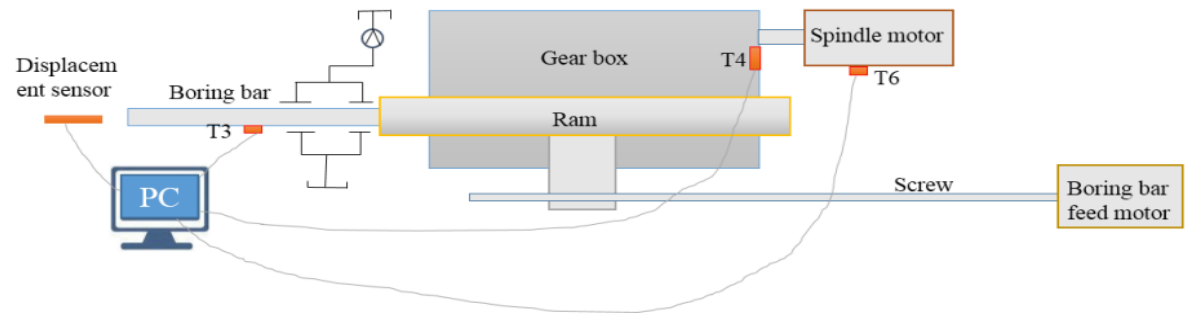

Figure 2. The boring bar structure diagram

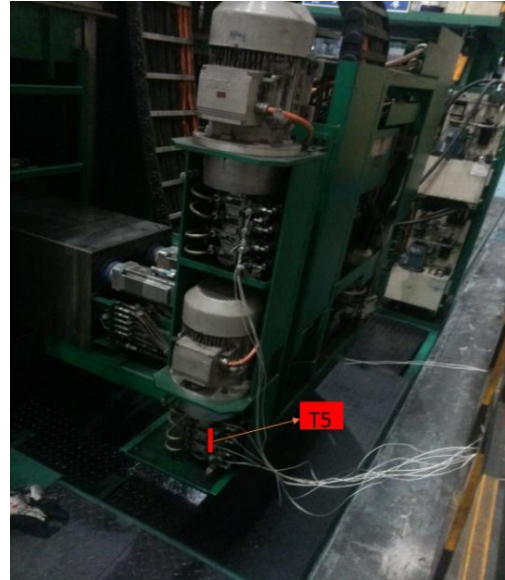

(a) Temperature sensor of $\mathrm{T} 5$

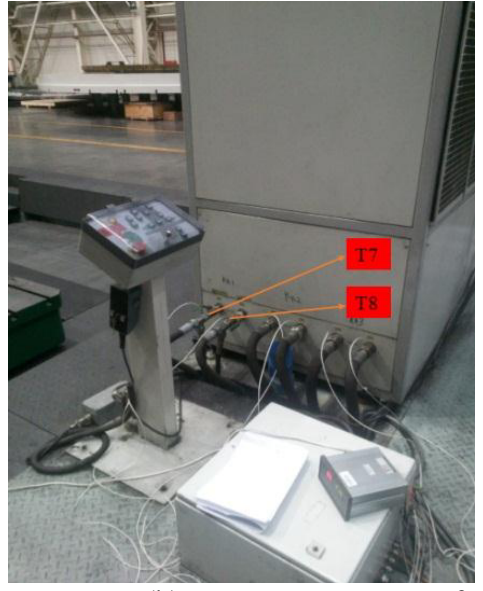

(b)Temperature sensor of $\mathrm{T} 7$ and $\mathrm{T} 8$

Figure 3. Installation of instrument

Table 1. The arrangement of 11 sensor locations

\begin{tabular}{|l|l|}
\hline T2, T5 & The boring bar hydrostatic oil temperature and hydrostatic pump location \\
T3, T4 & Front of the boring bar, gear box position \\
T9, T10, T11 & Up and down of boring bar bearing end and after boring bar bearing position \\
T7, T8, T6 & $\begin{array}{l}\text { Boring bar static pressure oil outlet and inlet position and the main shaft motor position } \\
\text { T1 }\end{array}$ \\
\hline
\end{tabular}

Table 2. Partial temperature data

\begin{tabular}{|c|c|c|c|c|c|c|c|c|c|c|}
\hline T1 & T2 & T3 & T4 & T5 & T6 & T7 & T8 & T9 & T10 & T11 \\
\hline 23.8 & 22.2 & 23.7 & 26 & 39 & 27 & 20.9 & 18.7 & 35.6 & 36.9 & 38.4 \\
\hline 23.3 & 21.3 & 24.4 & 25.3 & 40 & 27 & 22.6 & 18.8 & 33.6 & 35.6 & 37.1 \\
\hline 23.2 & 21.2 & 23.7 & 26.6 & 42 & 75 & 22.2 & 17.1 & 34.5 & 36.1 & 38.1 \\
\hline 23 & 21.1 & 23.3 & 29 & 43 & 81 & 21.2 & 16.2 & 31.8 & 33.6 & 35.1 \\
\hline 22.8 & 21.1 & 23.8 & 33.2 & 44 & 90 & 21.3 & 21.3 & 29.7 & 31.1 & 34.6 \\
\hline 22.7 & 21.1 & 24.3 & 36.5 & 44 & 96 & 21.9 & 22 & 29.1 & 30.1 & 34.2 \\
\hline 22.6 & 21 & 24.6 & 38 & 45 & 101 & 22.7 & 21.7 & 31.4 & 31.6 & 35.1 \\
\hline 22.5 & 20.9 & 23.8 & 40.5 & 46 & 104 & 22.1 & 17 & 35.1 & 35.6 & 36.4 \\
\hline 22.4 & 20.9 & 23.2 & 41.6 & 47 & 106 & 21.1 & 16.1 & 35.9 & 35.8 & 36.2 \\
\hline 22.2 & 20.8 & 23.4 & 43.3 & 47 & 108 & 20.9 & 20.8 & 29.4 & 30.8 & 33.3 \\
\hline 22.1 & 20.8 & 23.8 & 44 & 46 & 109 & 21.4 & 21.5 & 29.3 & 30.2 & 33.4 \\
\hline 22 & 20.7 & 24 & 45.3 & 47 & 110 & 22.3 & 19.8 & 31.7 & 32.2 & 35.1 \\
\hline 22 & 20.7 & 23.2 & 45.7 & 47 & 111 & 21.6 & 16.4 & 35.3 & 35.6 & 36 \\
\hline 22 & 20.7 & 22.8 & 46.2 & 47 & 112 & 20.7 & 20.2 & 31.1 & 31.5 & 33.1 \\
\hline 22 & 20.6 & 23.6 & 46.8 & 47 & 113 & 21.2 & 21.3 & 29.7 & 30.6 & 35.6 \\
\hline 22 & 20.6 & 24 & 47.2 & 47 & 113 & 22.2 & 21.9 & 30.1 & 30.4 & 33.3 \\
\hline 22 & 20.6 & 23.2 & 47.5 & 47 & 113 & 21.8 & 16.6 & 35.2 & 35.6 & 36.1 \\
\hline 22 & 20.6 & 22.7 & 47.9 & 48 & 114 & 20.9 & 18.6 & 31.8 & 32.4 & 33.2 \\
\hline 22.1 & 20.5 & 23.4 & 48.2 & 47 & 114 & 21.2 & 21.1 & 30.1 & 30.8 & 33.5 \\
\hline 22.1 & 20.5 & 23.7 & 48.4 & 47 & 114 & 21.9 & 22 & 29.6 & 30.7 & 33.7 \\
\hline 22.2 & 20.5 & 23.4 & 48.7 & 48 & 115 & 22 & 17.1 & 35 & 35.6 & 36.7 \\
\hline 22.2 & 20.5 & 23 & 48.8 & 48 & 114 & 21.1 & 16 & 36.3 & 36.1 & 36.3 \\
\hline 22.3 & 20.5 & 23.2 & 49.1 & 48 & 115 & 21.1 & 21 & 30.7 & 31.4 & 33.6 \\
\hline
\end{tabular}


In this experiment, stick out length of boring bar was set as $60 \mathrm{~cm}$, a dial indicator was installed at the front of the boring bar to measure the boring bar elongation. The inlet oil temperature and the oil return temperature of the static pressure radial bearing and the temperature of the sensor were measured by the temperature sensor Pt100. Temperature and the corresponding thermal elongation error data is recorded at every five minutes. The changing curves of temperature data gotten at each measuring point are shown in Fig. 4, and part data are shown in Table 2.

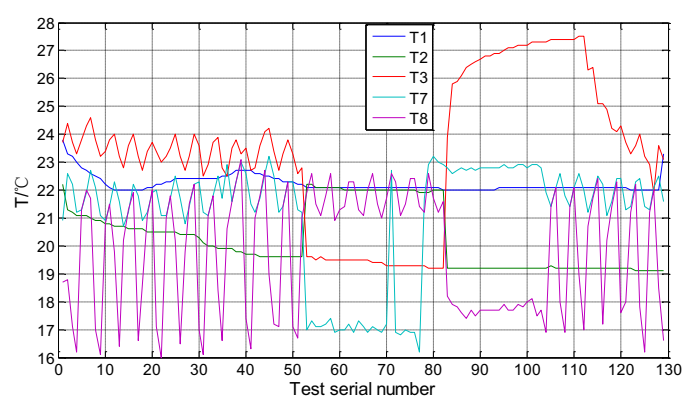

(a) Measured data curve of T1, T2, T3, T7, T8

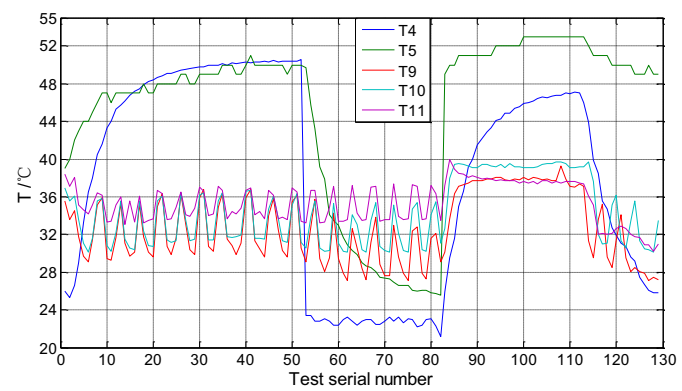

(b) Measured data curve of T4, T5, T9, T10, T11

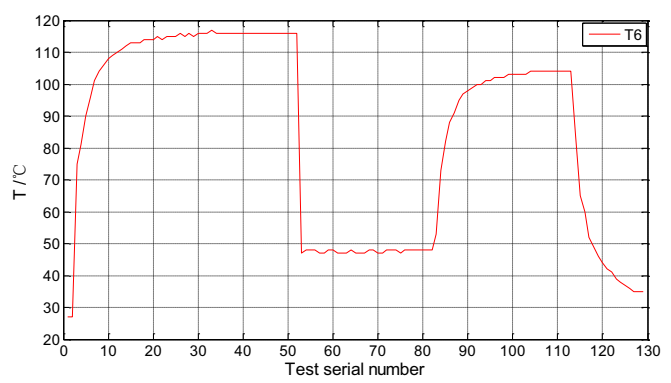

(c) Measured data curve of T6

Figure 4. Measured data of all temperature sensor

\subsection{Extraction the principal component of the temperature data by PCA}

130 sets of data were collected at every five minutes during the experiment, and so a $130 * 11$ temperature matrix can be established. By the method in Section 2, 11 principal component contribution rate are extracted and the cumulative contribution rates are shown Table 3. From the Table 3, it can be seen that the cumulative contribution rates of the first 4 principal components are reached $88.7835 \%$, so the number of main components is determined as 4 .

Table 3. Principal component calculation

\begin{tabular}{|c|l|c|}
\hline $\begin{array}{l}\text { Principal } \\
\text { component }\end{array}$ & $\begin{array}{l}\text { Variance } \\
\text { contribution } \\
\text { rate\% }\end{array}$ & $\begin{array}{c}\text { Cumulative } \\
\text { variance } \\
\text { contribution rate }\end{array}$ \\
\hline 1 & 53.2409 & 53.2409 \\
2 & 16.7726 & 70.0135 \\
3 & 10.6200 & 80.6335 \\
4 & 8.1500 & 88.7835 \\
5 & 4.7514 & 93.5349 \\
6 & 3.2069 & 96.7418 \\
7 & 0.9729 & 97.7147 \\
8 & 0.7847 & 98.4994 \\
9 & 0.7600 & 99.2594 \\
10 & 0.5768 & 99.8362 \\
11 & 0.1638 & 100.0000 \\
\hline
\end{tabular}

According to the calculation of the Table 3, the feature vector $\boldsymbol{C}=\left(\boldsymbol{\alpha}_{1}, \boldsymbol{\alpha}_{2}, \boldsymbol{\alpha}_{3}, \boldsymbol{\alpha}_{4}\right)$ of the principal component $\left.\boldsymbol{X}=\left(t_{1}, t_{2}, t_{3}, t_{4}\right)\right)$ is obtained, which is shown in Table 4 .

Table 4. Feature vector of principal components

\begin{tabular}{|r|r|r|r|}
\hline $\mathrm{a}_{1}$ & \multicolumn{1}{|c|}{$\mathrm{a}_{2}$} & \multicolumn{1}{c|}{$\mathrm{a}_{3}$} & \multicolumn{1}{c|}{$\mathrm{a}_{4}$} \\
\hline 0.0253 & -0.1065 & 0.7499 & 0.5480 \\
\hline-0.3338 & 0.2409 & 0.3576 & -0.1725 \\
\hline 0.3777 & -0.0665 & -0.1703 & 0.0813 \\
\hline 0.3232 & -0.2946 & 0.2553 & -0.3712 \\
\hline 0.3496 & -0.2782 & -0.1306 & 0.0714 \\
\hline 0.2962 & -0.2049 & 0.3673 & -0.4936 \\
\hline 0.3099 & -0.2516 & -0.0517 & 0.2529 \\
\hline-0.2711 & -0.1956 & -0.0246 & -0.4152 \\
\hline 0.3321 & 0.3911 & 0.0623 & -0.0867 \\
\hline 0.3116 & 0.4248 & -0.1255 & 0.0547 \\
\hline 0.2323 & 0.5339 & 0.2017 & -0.1727 \\
\hline
\end{tabular}

The extracted principal components can be expressed as follows:

$$
\begin{aligned}
& t_{1}=0.0253 T 1-0.3338 T 2+0.3777 T 3+0.3232 T 4+0.3496 T 5+0.2962 T 6+0.3099 T 7-0.2711 T 8+0.3321 T 9+0.3116 T 10+0.2323 T 11 \\
& t_{2}=-0.1065 T 1+0.2409 T 2-0.0665 T 3-0.2946 T 4-0.2782 \mathrm{~T} 5-0.2049 \mathrm{~T} 6-0.2516 \mathrm{~T} 7-0.1956 \mathrm{~T} 8+0.3911 \mathrm{~T} 9+0.4248 T 10+0.5339 T 11 \\
& t_{3}=0.7499 T 1+0.3576 T 2-0.1703 T 3+0.2553 T 4-0.1306 T 5+0.3673 T 6-0.0517 T 7-0.0246 T 8+0.0623 T 9-0.1255 T 10+0.2017 T 11 \\
& t_{4}=0.5480 T 1-0.1725 T 2+0.0813 T 3-0.3712 T 4+0.0714 T 5-0.4936 T 6+0.2529 T 7-0.4152 T 8-0.0867 T 9+0.0547 T 10-0.01727 T 11
\end{aligned}
$$




\subsection{Prediction of thermal extension error by LS-SVM}

As written as in Section 3, the LS-SVM model can be expressed as

$$
f(x)=\sum_{i=1}^{n} \boldsymbol{\alpha}_{i} K\left(x, x_{i}\right)+b
$$

The four temperature sensitive point is used as the input of the model.

$$
x=\left[T_{3}, T_{4}, T_{6}, T_{9}\right]
$$

The temperature matrix corresponding to the $i$

$$
x_{i}=\left[T_{3}^{i}, T_{4}^{i}, T_{6}^{i}, T_{9}^{i}\right]
$$

Among them $, j=(1,2, \cdots, l), l=130$. Before using the Gauss radial basis function as the kernel function of LS-SVM to carry on the training model, the value of the kernel parameter and the regularization parameter $S$ need to be determined. For the choice of these two parameters, directly determines the accuracy of the model, and they are determined to belong to the optimal model selection problem, so far there is no unified principle. In paper, by continuously adjusting the value of the two parameters, the combination of the minimum error of cross validation is selected as the best value of the parameters. Then, it can be obtained that $\sigma=20.3, s=0.085, b=0.239, l=130$ And, the prediction results of LS-SVM thermal error model are shown in Fig. 5.

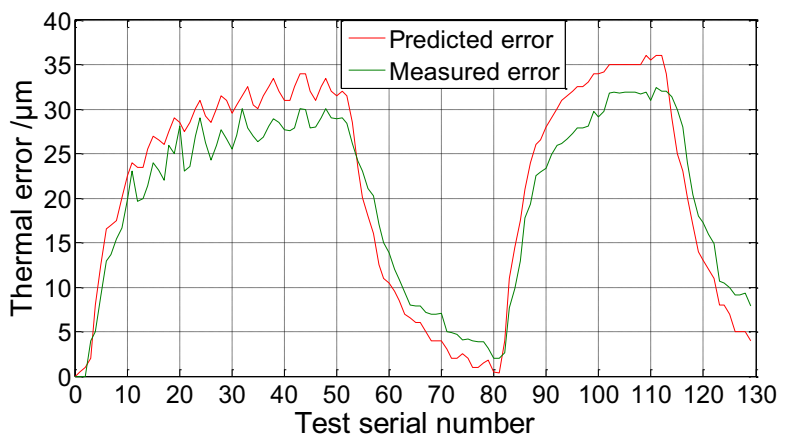

(a) Comparison of measured error and predicted error

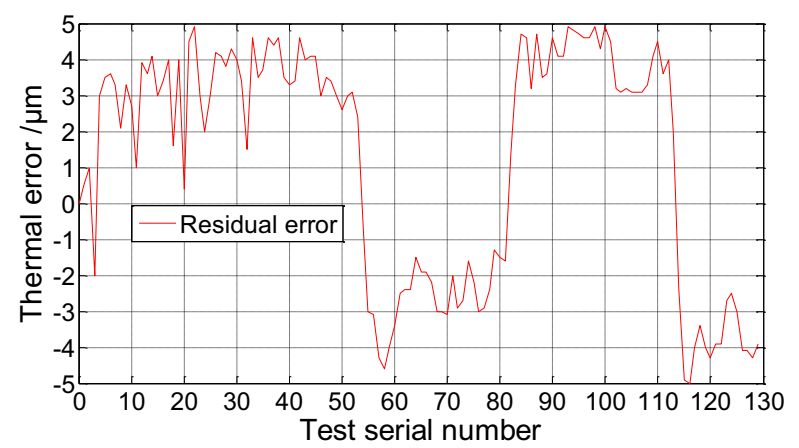

(b) Residualerror

Figure 5. Prediction results of LS-SVM method
By the method proposed in this paper, the results show that the residual value of the model is small, and the maximum error is only $4.9 \mu \mathrm{m}$ just as shown in Fig. 5. However, the maximum residual error gotten by the multiple linear regression model is $7.5 \mu \mathrm{m}$, which is shown in Fig. 6. Therefore, it is feasible to optimize the temperature measuring points by using the PCA and the LS-SVM method, and the accuracy and robustness of the model are remarkable.

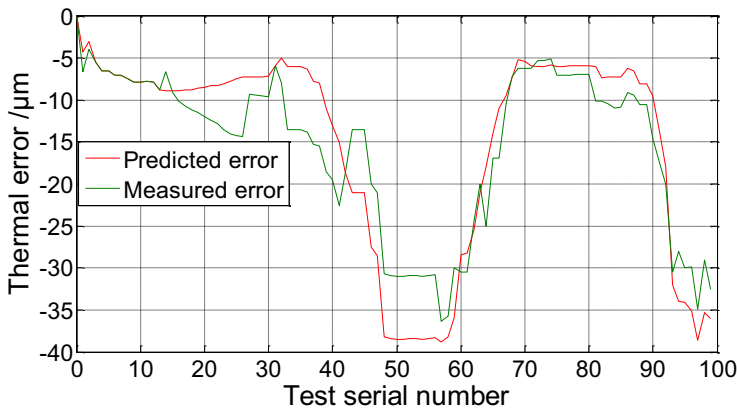

(a) Comparison of measured error and predicted error

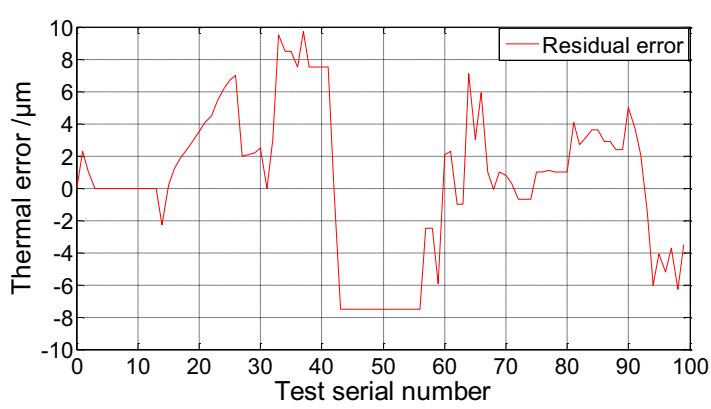

(b) Residualerror

Figure 6. Prediction results of multiple linear regression method

\section{Conclusions}

Thermal errors are the major contributor to the dimensional errors of a work-piece in precision machining. Error compensation technique is a cost-effective way to reduce thermal errors, but accurate modeling of errors is a prerequisite of error compensation. To this aim, in this paper, a method of PCA is proposed to select the optimization data of temperature measurement points, and SVM is introduced to predict thermally induced extension error of boring machine. Conclusions of this research are summarized as follows.

(1) By the method of PCA the dimension of temperature data from 11 down to 4 .

(2) LS-SVM are used to predict the thermally induced thermal extension error of boring bar, and the prediction accuracy of LS-SVM is superior to that of traditional model after comparison. Boring bar residual errors of thermal elongation down to $5 \mu \mathrm{m}$ or less, and the prediction can reduce the influence of unpredictable noises.

This method can effectively reduce the dimension of the temperature measuring points data and improve the robustness of the error needed in compensation model. Next, how to realize the real-time thermal error compensation efficiently will be the future research focus. 


\section{Acknowledgement}

The authors are most grateful to National Natural Science Foundation of China(51575010), The Leading Talent Project of Guangdong Province, Beijing Nova Program (Z151100000- 3150138), Shantou Light Industry Equipment Research Institute of Science and Technology Correspondent Station (2013B0909- 00008), Open Research Fund of Key Laboratory of High Performance Complex Manufacturing, Central South University (Kfkt2014-09),Basic Research Foundation of Beijing University of Technology, which they support the research presented in this paper.

\section{References}

1 Cheng Q, Luo R, Gu P, et al. Load Induced Error Identification and Camber Curve Design of a Large-Span Crossbeam[J]. Advances in Mechanical Engineering, 2013, 5: 847194.

2 Mehrabi M G, O'Neal G, Min B K, et al. Improving machining accuracy in precision line boring[J]. Journal of Intelligent Manu- facturing, 2002, 13(5): 379-389.

3 Bryan J. International status of thermal error research (1990)[J]. CIRP AnnalsManufacturing Technology, 1990, 39(2): 645-656.

4 Mayr J, Jedrzejewski J, Uhlmann E, et al. Thermal issues in machine tools[J]. CIRP Annals-Manufacturing Technology, 2012, 61(2): 771-791.

5 Postlethwaite S R, Allen J P, Ford D G. The use of thermal imaging, temperature and distortion models for machine tool thermal error reduction[J]. Proceedings of the Institution of Mechanical Engineers, Part B: Journal of Engineering Manufacture, 1998, 212(8): 671-679.

6 Ni J. CNC machine accuracy enhancement through real-time error compensation[J]. Journal of manufacturing science and engineering, 1997, 119(4B): 717-7

7 Yang S, Yuan J, Ni J. The improvement of thermal error modeling and compensation on machine tools by CMAC neural network[J]. International Journal of Machine Tools and Manufacture, 1996, 36(4): 527-537.

8 Kim H S, Jeong K S. Design and manufacture of a three-axis ultra-precision $\mathrm{CNC}$ grinding machine[J]. Journal of Materials Processing Technology, 1997, 71(2): 258-266.

9 Ramesh R, Mannan M A, Poo A N. Error compensation in machine tools - a review: Part II: thermal errors $[\mathrm{J}]$. International Journal of Machine Tools and Manufacture, 2000, 40(9): 1257-1284.

10 Mayr J, Jedrzejewski J, Uhlmann E, et al. Thermal issues in machine tools $[\mathrm{J}]$. CIRP Annals-Manufacturing Technology, 2012, 61(2): 771-791.
11 Zhao HT, et al. Selection method of temperature critical point based on thermal error sensitivity chart. Journal of Shanghai Jiaotong University, 2015,49(005): 725-729.

12 Wang XL, et al. Thermal error modeling of machine tool spindle based on PSO clustering and ELM neural network $[\mathrm{J}]$. Combination machine tools and automatic processing technology, 2015 (7): 69-73.

13 Luo FJ,Song DL, et al.Optimization of the Temperature Measuring PointsBased on LSR. Modular Machine Tool \&Automatic Manufacturing Technique ,2015,03(3):56-58.

14 Ma Y. Sensor placement optimization for thermal error compensation on machine tools[M]. University of Michigan., 2001.

15 Lee J H, Yang S H. Statistical optimization and assessment of a thermal error model for $\mathrm{CNC}$ machine tools[J]. International Journal of Machine Tools and Manufacture, 2002, 42(1): 147-155.

16 Creighton E, Honegger A, Tulsian A, et al. Analysis of thermal errors in a high-speed micro-milling spindle $[\mathrm{J}]$. International Journal of Machine Tools and Manufacture, 2010, 50(4): 386-393.

17 Han J, Wang L, Cheng N, et al. Thermal error modeling of machine tool based on fuzzy c-means cluster analysis and minimal-resource allocating networks[J]. The International Journal of Advanced Manufacturing Technology, 2012, 60(5-8): 463-472.

18 Cheng Q, Qi Z, Zhang G, et al. Robust modelling and prediction of thermally induced positional error based on grey rough set theory and neural networks $[\mathrm{J}]$. The International Journal of Advanced Manufacturing Technology, 2015: 1-12.

19 Zhao C, Gao F, Wang F. Nonlinear Batch Process Monitoring Using Phase-Based Kernel-Independent Component Analysis- Principal Component Analysis (KICA- PCA)[J]. Industrial \& engineering chemistry research, 2009, 48(20): 9163-9174.

20 Zhao C H, Wang F L, Lu N Y, et al. Stage-based Soft-transition Multiple PCA Modeling and on-line Monitoring Strategy for Batch Processes [J]. Journal of Process Control, 2007, 17(9) : 728 -741 .

21 Suykens J A K, Vandewalle J. Recurrent least squares support vector machines[J] . IEEE transactions on circuits and systems-I : Fundamental theory and applications, 2000, 47(7): 1109-1114.

22 Duan K, Keerthi S S, Poo A N. Evaluation of simple performance measures for tuning SVM hyper-parameters[J]. Neurocomputing, 2003, 51: 41-59.

23 Miao E M, Niu P C, Fei Y T, et al. Selecting temperature-sensitive points and modeling thermal errors of machine tools $[\mathrm{J}]$. Journal of the Chinese Society of Mechanical Engineers, 2011, 32(6): 559-565. 